\title{
Levitated Liquid Dynamics in Reduced Gravity and Gravity-Compensating Magnetic Fields
}

\author{
VALDIS BOJAREVICS ${ }^{1,3}$ and ROBERT W. HYERS ${ }^{2}$ \\ 1.-University of Greenwich, Park Row, London SE10 9LS, UK. 2.-University of Massachusetts, \\ Amherst, MA 01003, USA. 3.—e-mail: V.Bojarevics@greenwich.ac.uk
}

Dynamic models are used to investigate the behavior of liquid droplets suspended in alternating current and direct current magnetic fields in microgravity and in various configurations providing conditions similar to microgravity. The realistic magnetic fields of solenoidal coils are used for the modeling experiments with electrically conducting (liquid silicon or metal) droplets. At high values of magnetic field, some oscillation modes are damped quickly, while others are modified with a considerable shift of the oscillating droplet frequencies and the damping constants from the nonmagnetic case. On a larger scale, the models are used to investigate the melting and heating process of reactive materials. It is demonstrated how $1 \mathrm{~kg}$ of liquid titanium in a traditional "cold" crucible-type furnace can be fully levitated without contact to wall to achieve high superheat of the melt.

\section{INTRODUCTION}

From the electromagnetic (EM) levitation experiments ${ }^{1}$ in an alternating current (AC) magnetic field, it became apparent that the levitated liquid metal is prone to oscillation and instability. The EM and electrostatic levitation experiments with liquid metal droplets show difficulties related to confinement stability and a need for complex correction functions to establish a correlation between the measurements and the droplet material properties. ${ }^{2,3}$ The observed intense internal fluid flow is in the turbulent regime for earthbound conditions. A combination of AC and direct current (DC) magnetic fields was recently recognized as efficient tool for the magnetically stabilized treatment of molten substances without a contact to contaminating walls. ${ }^{4,5}$ The intense AC magnetic field required to produce levitation results in turbulent large-scale toroidal recirculation, while the use of a homogenous DC magnetic field, allows the toroidal flow to be damped. However, the magnetically modified turbulence could make the effective viscosity behave in a nonlinear fashion depending on the DC and AC magnetic field intensity. ${ }^{6}$ The flow in typical melting conditions is approaching the conditions with laminar viscosity and heat transfer when a uniform DC magnetic field exceeds about 4-5 T. In addition to the modified damping, the electrically conducting liquid droplet in a high DC magnetic field behaves quite differently to the nonconducting one: some oscillation modes are damped completely, and other modes are persisting for long times or damped moderately. ${ }^{p}$

On a larger scale, the magnetic suspension of liquid reactive materials, for example titanium alloys, can be used for high-quality castings. However, it is often difficult to achieve the required superheat in the melt with traditional "cold" crucible-type furnaces due to a partial contact with the water-cooled copper walls. ${ }^{8}$ If the contact is avoided, then thermal losses would be limited only by radiation and possible evaporation. This would produce a higher superheat and permit investigation of materials at extreme temperatures. At the other extreme, a highly undercooled liquid can be obtained before solidification to a glassy structure in the levitated conditions in absence of nucleation centres. Existing experimental evidence suggests that it is possible to melt and levitate several kilograms of liquid metal. ${ }^{9}$ In numerical experiments, ${ }^{10}$ it is demonstrated that full levitation of the liquid metal is achievable but requires careful optimization of the EM force to generate intense tangential flow along the surface away from the bottom stagnation point. 


\section{MAGNETIC FORCE ANALYSIS}

The common approach for theoretical models attempting to predict the free surface behavior of magnetically levitated droplets is based on the idea of an infinitely thin skin layer where the highfrequency $\mathrm{AC}$ magnetic field penetrates. This permits to replace the EM force volume distribution by the so-called "magnetic pressure" acting on the free surface. Such an approximation effectively removes any dependence on the electrical conductivity of the levitated material. The skin-layer approximation was tested against a direct numerical solution ${ }^{11}$ for the typical experimental frequencies and material properties ( $\mathrm{Al}, \mathrm{Ni}$, and $\mathrm{Si})$. The results indicate that for medium frequencies $(<100 \mathrm{kHz})$, the deformation and velocity field are significantly different for a 1-cm size droplet compared to the asymptotic skinlayer case. For higher frequencies $(>100 \mathrm{kHz})$, a relatively good correspondence for the droplet shape can be obtained. The velocity field within a levitated liquid material is controlled by the EM force volumetric distribution; therefore, it is important to understand how various approximations to this force field can be obtained from a mathematical point of view. The general expression for EM force acting on a levitated fluid volume is rather complex $^{12}$ if accounting for variable electrical conductivity and magnetic properties. For uniform magnetic properties within the liquid, the commonly used expression for the force is

$$
\mathbf{f}=\mathbf{j} \times \mathbf{B}+\chi \nabla\left(B^{2} / 2 \mu_{0}\right),
$$

where $\mathbf{j}$ is the electric current density, $\mathbf{B}$ is the magnetic field, $\chi$ is the volumetric magnetic susceptibility (e.g., $\chi=-4.2 \times 10^{-6}$ for silicon, which is diamagnetic), and $\mu_{0}$ is the magnetic permeability of vacuum in SI units. When a combination of highfrequency $\mathrm{AC}$ and $\mathrm{DC}$ magnetic fields is used, a time average of the force over the field oscillation period $\tau$ $\left(\sim 10^{-4} \mathrm{~s}\right.$ to $\left.10^{-6} \mathrm{~s}\right)$ is effective at the time scale of typical levitated droplet oscillation period $10^{\circ}-10^{-2}$ Hz. However, the oscillating part of the highfrequency $\mathrm{AC}$ field is still present within the fluid, adding a time-dependent vibrating force at double the frequency of the applied AC, in addition to the time-averaged force.

In the pseudo-spectral code SPHINX, ${ }^{6,8,10}$ the EM force is computed from an integral equation representation. This has an advantage that the boundary conditions are not explicitly required and the EM field can be solved only in the regions where it is needed. The electric current distribution in a moving medium of conductivity $\sigma$ is then given by the magnetic vector potential $\mathbf{A}$, the magnetic field $\mathbf{B}=\nabla \times \mathbf{A}$, the electric potential $\varphi$, and the fluid flow-induced part $\sigma \mathbf{v} \times \mathbf{B}$.

$$
\mathbf{j}=\sigma\left(-\partial_{t} \mathbf{A}-\nabla \varphi+\mathbf{v} \times \mathbf{B}\right)=\mathbf{j}_{\mathrm{AC}}+\mathbf{j}_{\mathrm{v}}
$$

The $\mathbf{j}_{\mathrm{AC}}$ part of the current is induced in the conducting medium even in the absence of velocity. The governing integral equations can be obtained from the electric current distribution in the source coils and the unknown induced currents in the liquid related to the total magnetic field and the vector potential $\mathbf{A}$ by the Biot-Savart law. ${ }^{12,13}$

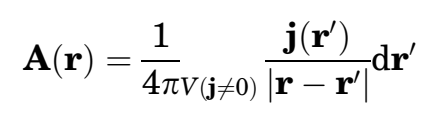

Equations 2 and 3 can be solved efficiently in the axisymmetric case for harmonic fields. ${ }^{13}$ The induced current in the liquid drop depends on its instantaneous free surface shape and needs to be recomputed as the shape changes during the oscillation development. The resulting EM force $\mathbf{f}$, timeaveraged over the AC period, similarly to Eq. 2, can be decomposed in two parts: $\mathbf{f}=\mathbf{f}_{\mathrm{AC}}+\mathbf{f}_{\mathrm{v}}$. The second, fluid velocity dependent part of the force $\mathbf{f}_{\mathrm{v}}$ can include DC and AC time-averaged contributions. Even in a pure AC field, in principle, there is a timeaveraged interaction with the more slowly varying velocity field.

The EM force associated to the electric current $\mathbf{j}=\left(\nabla \times \mathbf{B} / \mu_{0}\right)$ can be decomposed into the following two parts:

$$
\begin{aligned}
\mathbf{f}=\mathbf{j} \times \mathbf{B}=\left(\nabla \times \mathbf{B} / \mu_{0}\right) \times \mathbf{B}= & -\nabla\left(B^{2} / 2 \mu_{0}\right) \\
& +(\mathbf{B} \nabla) \mathbf{B} / \mu_{0}
\end{aligned}
$$

The first term on the right-hand side, being a gradient, is the "magnetic pressure," which can be incorporated into a modified pressure function

$$
p_{\mathrm{m}}=p+B^{2} / 2 \mu_{0}-\chi B^{2} / 2 \mu_{0}
$$

including also the force on magnetic material. For a diamagnetic material, $\chi<0$; the magnetic contribution gives an additional "compression" to the liquid droplet. However, unlike the induced current part, this magnetic force is not concentrated in the skin layer. The magnetic pressure function is used in the code SPHINX to modify the free surface boundary condition for the diamagnetic part of the force. The AC field-induced part is always solved explicitly within the fluid volume to account for both the rotational and the gradient parts in Eq. 4.

\section{THE NEED FOR REDUCED GRAVITY}

In electromagnetic levitation (EML) with $\mathrm{AC}$, the same forces that support the sample against gravity also stir the molten sample. As discussed in the next section, the flows in 1-g are invariably turbulent. The larger, kilogram-scale industrial samples have even higher turbulence intensity. However, in low gravity, the weaker positioning forces allow laminar flow for some conditions.

In two-frequency EMLs such as TEMPUS, ${ }^{14}$ or material science laboratory electromagnetic 
levitator (MSL-EML) ${ }^{15}$ the levitation field is the superposition of two components, each optimized for a different purpose. The positioning field has a large gradient but a low magnetic field. This combination results in a sufficient force with minimal heating of the sample. In contrast, the heating field has a very small gradient, so it produces almost no net force in the axial direction, although it does compress the equator of the sample. The heating field has a larger average magnetic field strength, resulting in larger induced currents and more Joule heating than the positioning field. The heating field also provides much more stirring than the positioning field for comparable currents.

There are many different classes of experiments performed in microgravity EML, each with a different reason for requiring reduced gravity. The key advantages of microgravity EML include low positioning forces, symmetric samples, lower minimum temperature than 1-g EML, and variable flow over a wide range, including both laminar and turbulent flow for most materials. For the measurement of surface tension and viscosity by the oscillating drop method, ${ }^{2,16}$ the low positioning forces allow nearspherical samples, improving the quality of the oscillations and thus the precision of the measurements. Also, the measurement of viscosity requires laminar flow in the sample without additional damping, only achievable in EML in microgravity. Experiments on phase selection ${ }^{17}$ require variable flow conditions to test theories about the effect of internal convection on the lifetime of metastable phases. Microgravity EML provides a much wider range of accessible flow than other methods (Fig. 1). Experiments on nucleation kinetics require laminar flow with a low-enough shear rate to allow time for diffusion-controlled nucleation. ${ }^{19}$ Finally, experiments on glass-forming alloys have the additional requirement of low-temperature processing, below the capabilities of 1-g EML.

Some of the advantages of reduced-gravity EML are available in the hybrid AC + DC pure DC levitation systems described in the following sections. The hybrid system superimposes a static magnetic field up to about $5 \mathrm{~T}$, greatly reducing fluid flow and forcing the flow to a laminar state. Such systems have been demonstrated to enable oscillation calorimetry to measure not only the specific heat but also thermal conductivity of levitated samples, ${ }^{4}$ otherwise feasible only in microgravity EML. On the other hand, as described below, the surface oscillations of drops in a hybrid levitator are very different than the free oscillations used to measure surface tension and viscosity. With a large applied DC field, the damping is primarily determined not by viscosity but by the interactions between the conducting sample and the applied field. The pure DC levitation system has no flow driven by the positioning field, but it requires a very large magnetic field, often more than $15 \mathrm{~T}$.

For experiments on solidification, nucleation, and phase selection, other complications from hybrid and DC levitation systems also come into play. While both systems can provide laminar flow, the utility of that situation depends on the particular materials system under study. A large applied magnetic field has been shown ${ }^{20}$ to shift phase equilibria and transformation kinetics in at least some systems. Such shifts can be problematic for extrapolating the results back to zero-field conditions.

\section{TRANSITION TO TURBULENCE IN EML}

Some classes of experiments, e.g., nucleation statistics in pure metals, ${ }^{21}$ may be unaffected by the internal flow in the samples. However, for other experiments described in the previous section, flow is an essential parameter. There even exist experiments in between, where the flow does not affect the experiment. For example, so long as the flow does not introduce additional damping of surface oscillations, the oscillation experiments are insensitive to the magnetohydrodynamic flow. In this case, it is important to keep the conditions far from the critical Reynolds number $R e_{\mathrm{c}}$.

Calculations show that flow in 1-g is always turbulent because of the strong forces needed to support a sample against gravity. In microgravity, both laminar and turbulent flows are possible. Experiments on an 8-mm diameter sample of $\mathrm{Pd}_{82} \mathrm{Si}_{18}$ used tracer particles to show the state of the flow. ${ }^{22} \mathrm{An}$ example video is available online. ${ }^{23}$ The sample was heated under constant magnetic conditions. As the temperature increased, the viscosity decreased, increasing the velocity of the internal flow quasistatically. The internal flow was calculated by a method similar to that described above to estimate the velocity as a function of temperature and time. As the sample melts, the tracers collect in the stagnation line at the equator. At a calculated
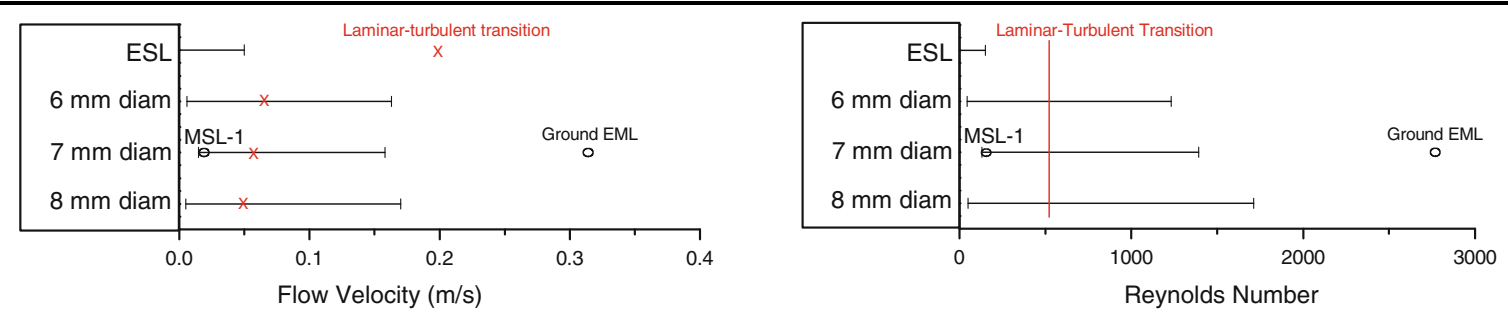

Fig. 1. Feasible ranges of velocity and Reynolds number achievable in ground-based and microgravity EML and ESL. ${ }^{18}$ 
Reynolds number of about 525, the flow becomes unsteady, with oscillations growing in magnitude and becoming nonlinear as the flow accelerates. Finally, the flow becomes chaotic at a calculated Reynolds number of about 600 . These flows are just above the transition from laminar flow and are neither isotropic nor fully developed. However, these flows are chaotic, show enhanced mixing, and definitely not laminar. For still faster flows, the turbulence continues developing; cf. Reynolds number of $10^{4}$ for the silicon droplet later in this article. Further observations of flow transitions in microgravity EML will enable refinement of the transitional Reynolds number.

\section{RESULTS FOR LIQUID SILICON DROPLET IN SOLENOID MAGNETS}

The following section gives some examples demonstrating (I) the dynamics of molten silicon droplet (diamagnetic and electrically conducting) when levitated in the high-frequency AC magnetic field and (II) the effect of adding the DC magnetic field in attempts to damp the surface oscillation and the internal turbulent velocity field. The pseudospectral code SPHINX ${ }^{6,8,10}$ is used to obtain the coupled EM and computational fluid dynamics solutions accounting for the magnetic force excited turbulent flow. The solution includes the turbulence damping by the high magnetic field both for the large-scale flow and for the small-scale turbulent properties. ${ }^{8}$

\section{AC + DC Field Levitation}

The numerical simulation starts with a preheated liquid silicon droplet of spherical shape (radius $R_{0}=5 \mathrm{~mm}$ ). The EM force from the interaction with the AC coil supports the droplet against gravity and deforms the shape due to the magnetic force distribution. The coil geometry (Fig. 2) is similar to the previous experimental and numerical investigation, ${ }^{4}$ where the finite-difference numerical model was applied for a fixed-shape (nonoscillating) spherical droplet and compared to the temperature field change when additional DC magnetic field was introduced. The solution in Ref. 4 was obtained only for the static magnetic field exceeding $4 \mathrm{~T}$, when the flow is sufficiently damped by the action of the magnetic field, and it did not permit to compute the flow at zero or moderate DC magnetic field due to the high-intensity internal flow in the droplet.

The droplet in experimental conditions is subject to a noticeable surface deformation and the intense turbulent flow in the droplet interior. These features are addressed by the use of the pseudospectral numerical solution. In the absence of an additional DC magnetic field, the computed flow is shown in Fig. 3. The temperature field achieves a steady-state distribution after cooling by radiation from the initial preheated stage of $1700^{\circ} \mathrm{C}$ and the following Joule heat input from the induction heating by the $\mathrm{AC}$ current redistributed by the

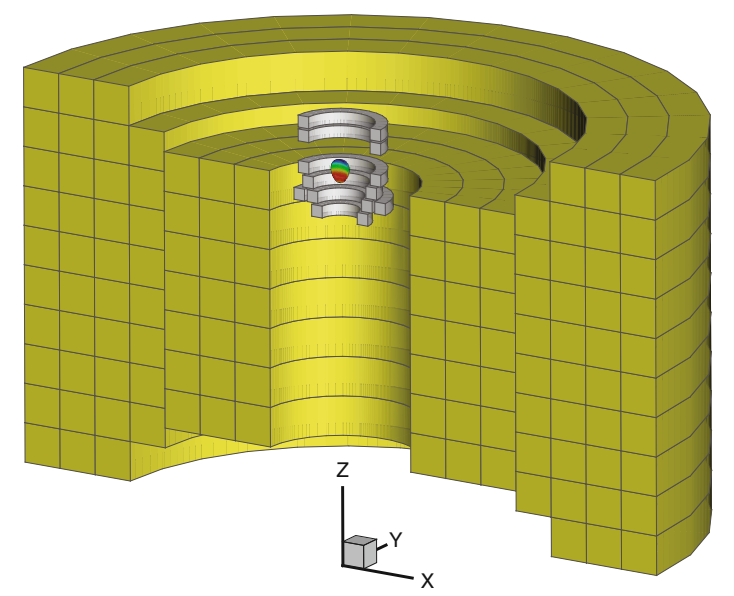

Fig. 2. Liquid silicon droplet $R_{0}=5 \mathrm{~mm}$ surrounded by the $\mathrm{AC}$ field coil positioned in the bore of DC solenoid magnet.

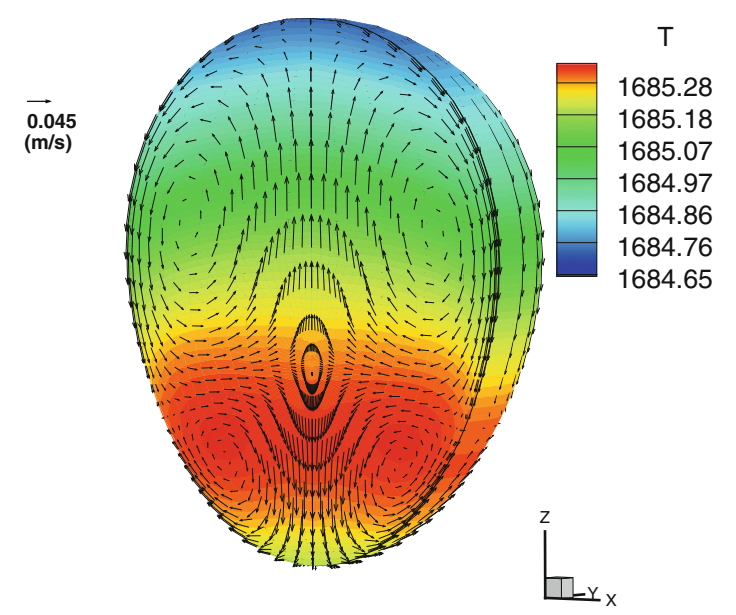

Fig. 3. Silicon droplet in the AC field (coil current 235 A) in the absence of DC field, view to instantaneous velocity and temperature field after $5 \mathrm{~s}$.

turbulent convection and diffusion. The nonpotential part of the time-averaged AC EM force creates an intense internal flow of typical magnitude of about $0.1 \mathrm{~m} / \mathrm{s}$, which for a liquid silicon droplet of $10-\mathrm{mm}$ diameter gives an estimate of a typical Reynolds number of the order $10^{4}$. This flow is mildly turbulent with the effective turbulent viscosity and the thermal diffusion enhanced by the action of the turbulence. Figure 4 demonstrates the effective viscosity distribution as computed from the $k-\omega$ model turbulence model. ${ }^{8}$ Figure 4 shows the violent oscillation pattern observable on the droplet surface $R_{\mathrm{t}}$ (top position) and the dominating oscillation up and down of the center of mass $Z_{\mathrm{t}}$ for the droplet as a whole. The solution accounts for the continuous adjustment of the EM field in the liquid during the development of these oscillations. Clearly, this type of liquid flow and oscillation act detrimentally to any attempts to measure material properties of the levitated liquid sample. 


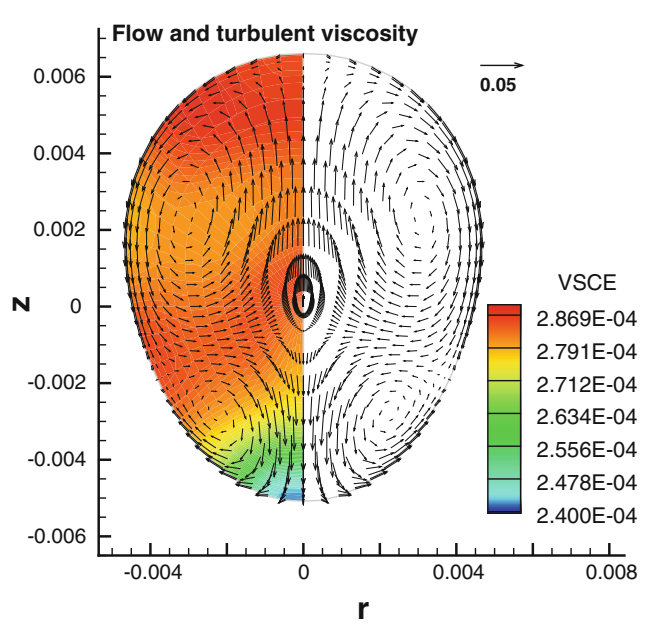

(a)

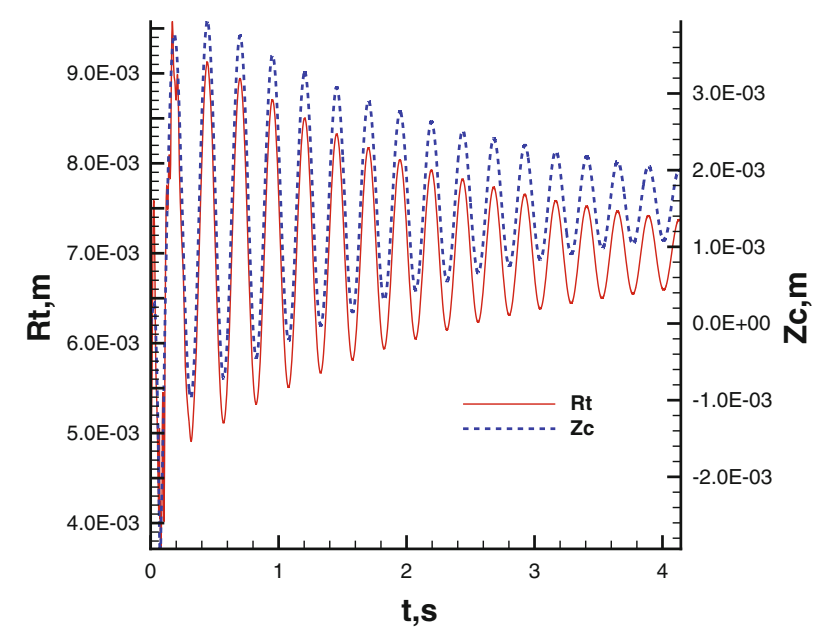

(b)

Fig. 4. (a) Velocity and turbulent viscosity distribution in the silicon droplet levitated in AC field in the absence of DC field. (b) The oscillation pattern for the droplet surface $R_{\mathrm{t}}$ and center of mass $Z_{\mathrm{c}}$ at $B_{\mathrm{dc}}=0$.

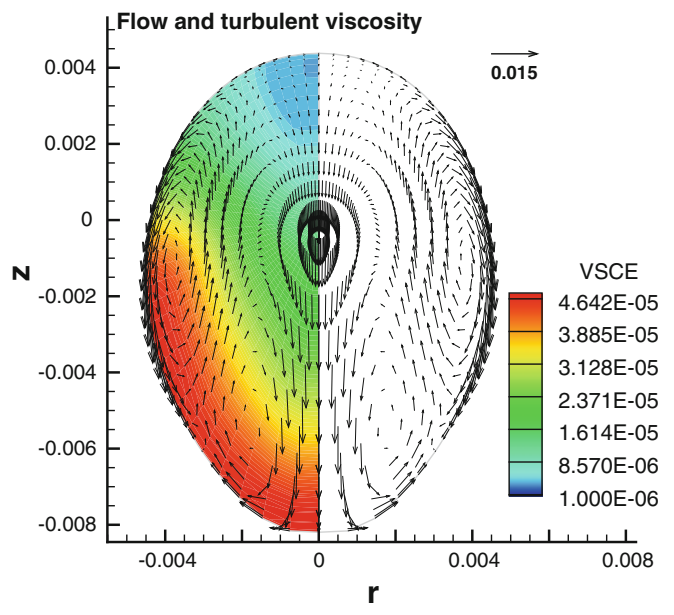

(a)

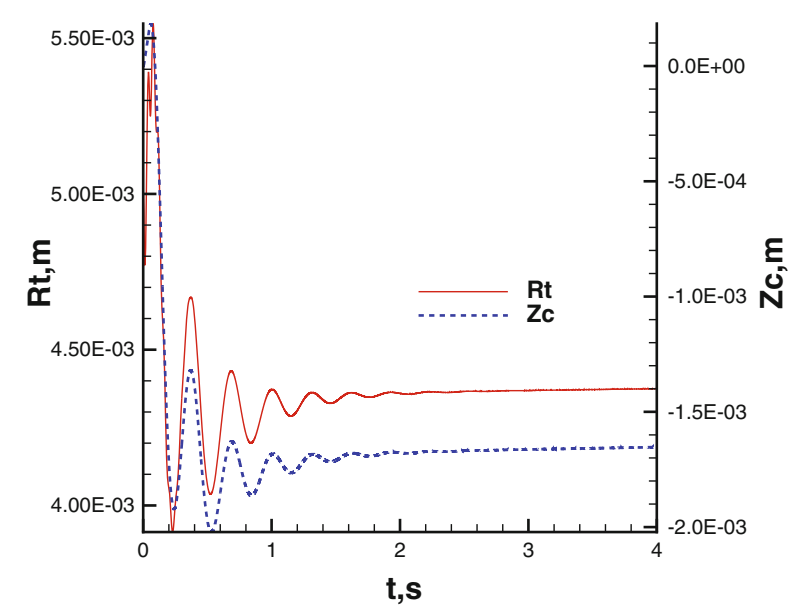

(b)

Fig. 5. (a) Velocity and turbulent viscosity distribution in the silicon droplet levitated in AC field with the addition of DC uniform field of 4 T. (b) The oscillation pattern for the droplet surface $R_{\mathrm{t}}$ and center of mass $Z_{\mathrm{c}}$ at $B_{\mathrm{dc}}=4 \mathrm{~T}$.

In attempts to damp the electromagnetically driven convective flow and the associated turbulent diffusion, the authors of Refs. 4 and 5 proposed to use a uniform vertical DC magnetic field. They observed in the experiment that a relatively large field of about $4 \mathrm{~T}$ is required to bring the droplet to conditions close to the laminar flow and thermal diffusion. Our model permits prediction of the flow behavior when the DC field is absent and then gradually increased. As can be seen from Fig. 5, at $B_{\mathrm{z}}=4 \mathrm{~T}$, the flow intensity decreases and the turbulent viscosity is significantly reduced. The droplet oscillation is quickly damped at this magnitude of the DC magnetic field. The flow is not completely laminar.

The flow becomes practically laminar when the DC magnetic field reaches $10 \mathrm{~T}$, as shown in Fig. 6 .
At the higher field values, the thermocapillary effects start to be noticeable as these are apparently in the direction parallel to the surface. The Marangoni convection loop forms at the top part of droplet where the temperature gradient is sufficiently large. The oscillations of the surface are strongly affected by the center of mass longitudinal motion along the DC magnetic field lines. Some modes of surface motion are modified in the presence of strong uniform field. ${ }^{6,7}$ The damping of this oscillation is relatively low and persists even at even higher magnetic fields.

\section{Field Levitation}

A purely DC magnetic levitation, using paramagnetic and diamagnetic properties of the materials, 


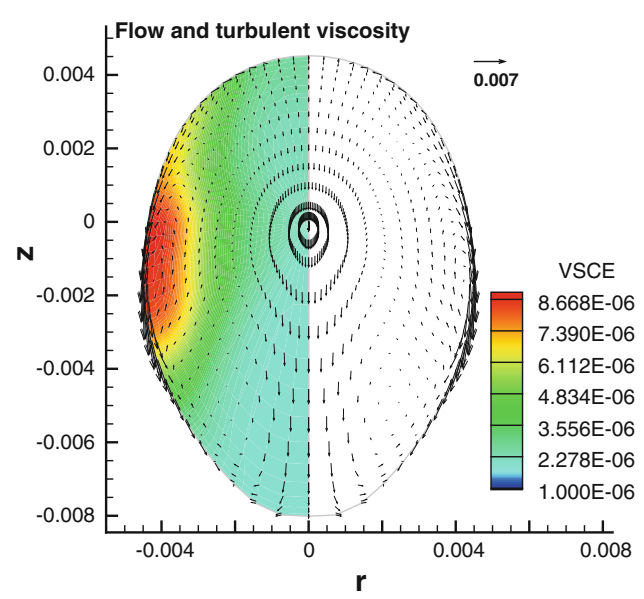

(a)

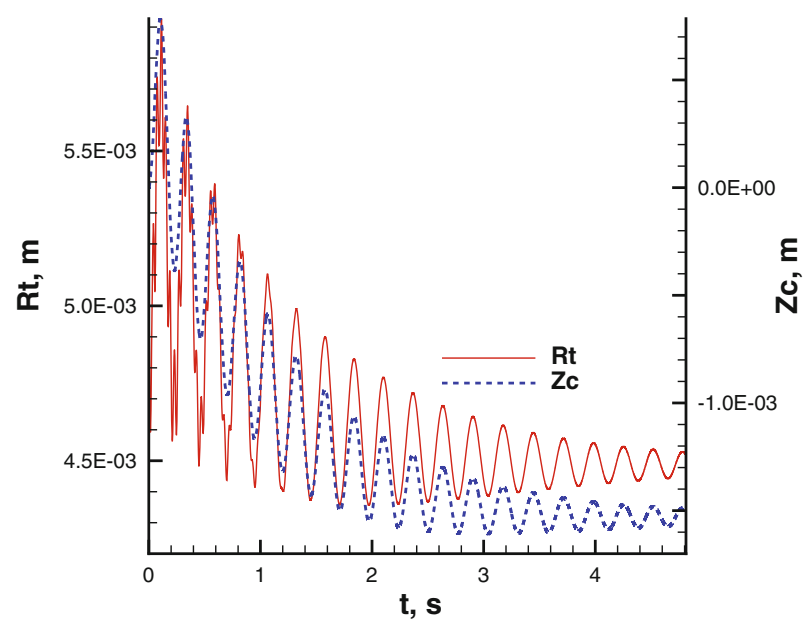

(b)

Fig. 6. (a) Velocity and turbulent viscosity distribution in the silicon droplet levitated in AC field with the addition of DC uniform field of $10 \mathrm{~T}$. (b) The oscillation pattern for the droplet surface $R_{\mathrm{t}}$ and center of mass $Z_{\mathrm{c}}$ at $B_{\mathrm{dc}}=10 \mathrm{~T}$.
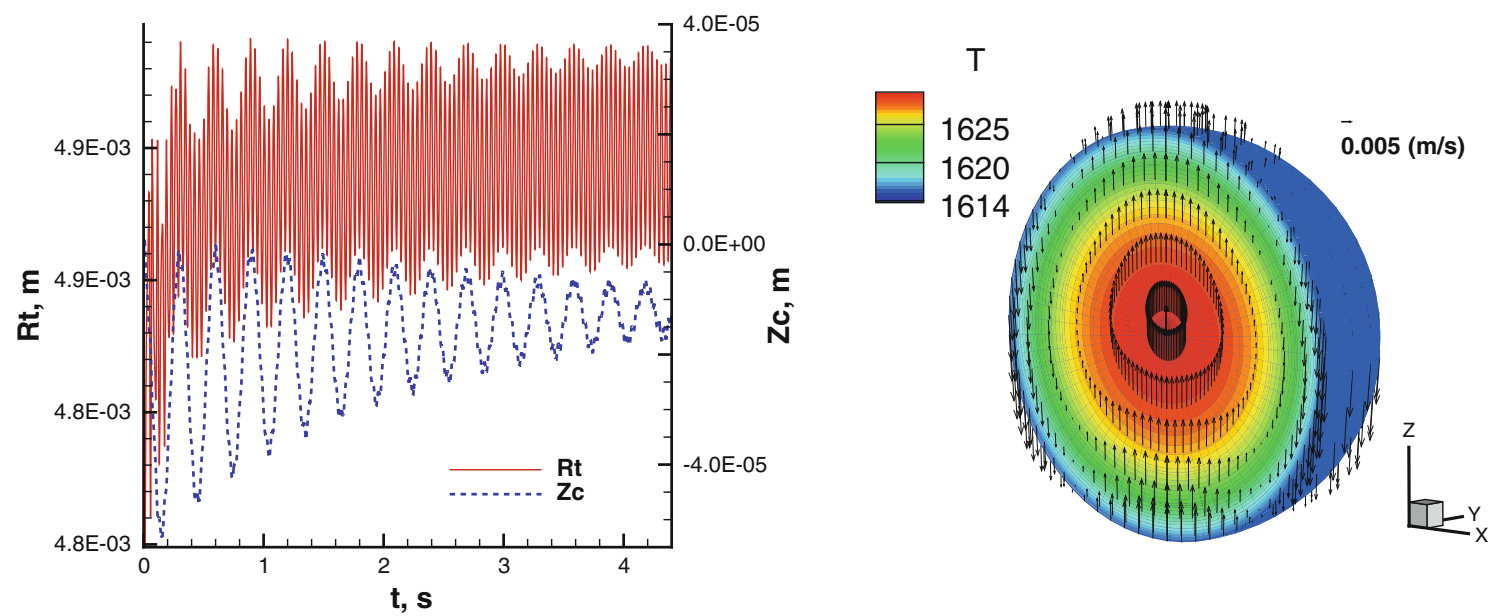

Fig. 7. Oscillations of the surface $R_{\mathrm{t}}$ and the center of mass $Z_{\mathrm{c}}$ for the liquid silicon droplet $R_{0}=5 \mathrm{~mm}$ in $17 \mathrm{~T}$ DC field at the top part of the solenoid coil.

can be realized for liquid materials if sufficiently large gradient fields are available (typically $>$ $10 \mathrm{~T}$ ). The vertical field gradient permits to compensate gravity along the central axis, while the radial variation opposes motion of the droplet and acts to center it on the axis for stable levitation (for diamagnetics). The motions of an electrically conducting levitated droplet are expected to be damped in a strong DC magnetic field; however, some types of movement are not affected in particular field configurations. In the microgravity case $(g=0)$ and a uniform vertical magnetic field, the asymptotic solution $^{7}$ shows that odd axisymmetric oscillation modes are very moderately damped and the frequencies are reduced significantly. It is not immediately clear if this behavior will stay in the presence of terrestrial gravity and the gradient magnetic field required for the magnetic levitation. The numerical experiments with liquid silicon appear to support the general conclusions of the asymptotic solution as demonstrated by Fig. 7 . The $L=3$ mode (see the velocity field in Fig. 7) is not damped immediately in the 17 T magnetic field required to levitate the $5 \mathrm{~mm}$ radius liquid silicon droplet. The frequency of the $L=3$ mode is reduced about $\sqrt{ } 3$ times relative to the nonmagnetic case and closely matches the theoretical value. ${ }^{7}$ The flow is purely laminar as can be seen from the lack of mixing that gives the radiationcooling-dominated temperature distribution in Fig. 7.

\section{LIQUID TITANIUM MELT IN COLD CRUCIBLE}

When levitating small droplets, the surface tension is a crucial effect preventing the liquid leaking 


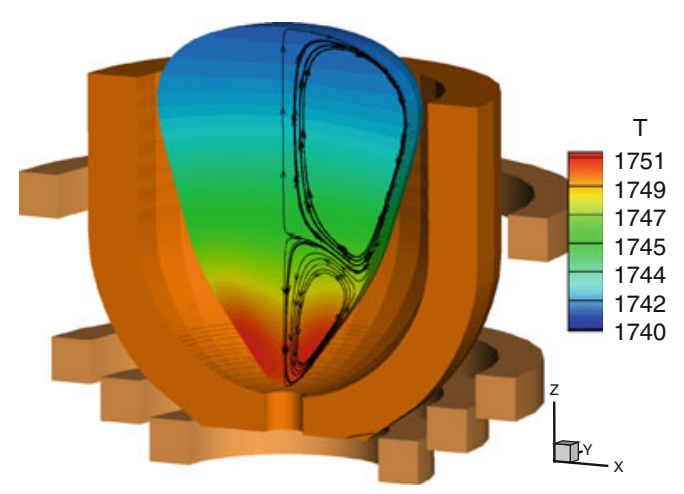

(a)

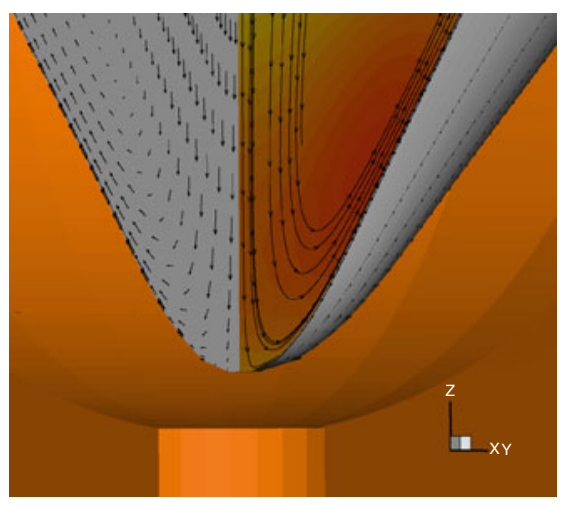

(b)

Fig. 8. (a) One kilogram of liquid titanium magnetically levitated in AC field after $10 \mathrm{~s}$ of the flow and shape adjustment to a quasi-stationary shape. (b) The zoom-in view shows the velocity field at the bottom.

at the bottom critical point where the AC EM force is locally zero due to symmetry condition. However, some experimental and theoretical evidence suggests that larger volumes of liquid metal can still be levitated in nearly axisymmetric AC magnetic field. The following solution for stably levitated $1 \mathrm{~kg}$ of liquid titanium was obtained using the AC frequency of $10 \mathrm{kHz}$ in the four-turn coil surrounding a suitably shaped crucible made of copper segments (often called "cold crucible" due to the internal water cooling of the segments). Starting from the initial hemispherical load, after a few seconds of the intense flow development, the numerically computed interface moves to a relatively stable position shown in Fig. 8. The total EM force acting on the fluid volume adjusts, approaching in an oscillating way the value of the total weight of the liquid metal. The bottom of the fluid initially is in contact with the solid wall, but in few hundred of milliseconds, it is pushed up, detaches, and after several oscillations, assumes the quasi-steady-levitated position. The zoom-in view of the bottom velocity field at this stage is shown in Fig. 8. The bottom shape continues a slight oscillation, readjusting the force balance.

The mechanism of the levitation, and particularly the local magnetic support at the bottom, appears to be dynamic in nature. ${ }^{10}$ The explanation for the fact that the liquid at the bottom is prevented from leaking and flowing down, is related to the particular velocity field in this region. The bottom vortex in Fig. 8 is maintained by the rotational nature of the EM force $\left(\operatorname{curl} \mathbf{f}_{\mathbf{e}} \neq 0\right)$, which drives the fluid tangentially upwards at the side surface of the liquid, away from the bottom stagnation point. Due to the continuity of the velocity field, the outflow at the bottom is redirected to the intense flow upwards along the side surface.

In the levitated conditions, the temperature of liquid metal rises relative to the case when there is a contact to the water-cooled copper crucible. The high heat exchange rates with the intense turbulence at the crucible wall are responsible for the temperature drop in a conventional crucible. The superheat relative to the titanium melting temperature $\left(1667^{\circ} \mathrm{C}\right)$ can easily exceed $100^{\circ}$ if the metal is fully levitated as shown in Fig. 8.

\section{CONCLUSIONS}

Numerical models give useful insight to the levitated liquid droplet dynamics at various combinations of magnetic fields in 1-g, reduced gravity, and a gravity-compensating force field. The more familiar EML in a high-frequency $\mathrm{AC}$ field can be improved by adding uniform DC magnetic field of an optimum magnitude to achieve nearly laminar flow conditions required for material property measurements. A high-intensity gradient DC field offers even better conditions to levitate diamagnetic liquid substances (conducting or nonconducting). A large volume of liquid metal can be fully levitated without contact to the wall to achieve high superheat of the melt in a specially designed, traditional "cold" crucible-type furnace.

\section{REFERENCES}

1. E. Okress, D. Wroughton, G. Comenetz, P. Brace, and J. Kelly, J. Appl. Phys. 23, 545 (1952).

2. I. Egry, G. Lohofer, I. Seyhan, S. Schneider, and B. Feuerbacher, Int. J. Thermophys. 20, 1005 (1999).

3. D.L. Cummings and D.A. Blackburn, J. Fluid Mech. 224, 395 (1991).

4. T. Tsukada, H. Fukuyama, and H. Kobatake, Int. J. Heat Mass Transf. 50, 3054 (2007).

5. H. Yasuda, Solidification of Containerless Undercooled Melts, ed. D.M. Herlach and D.M. Matson (Berlin, Germany: Wiley-VCH Verlag, 2012), pp. 305-320.

6. V. Bojarevics and K. Pericleous, CFD Modeling and Simulation in Materials, ed. L. Nastac, L. Zhang, B.G. Thomas, A. Sabau, N. El-Kaddah, A.C. Powell, and H. Combeau (Warrendale, PA: TMS, 2012), pp. 245-252.

7. J. Priede, J. Fluid Mech. 671, 399 (2010).

8. V. Bojarevics, R.A. Harding, K. Pericleous, and M. Wickins, Metall. Mater. Trans. B 35B, 785 (2004).

9. H. Tadano, M. Fujita, T. Take, K. Nagamatsu, and A. Fukuzawa, IEEE Trans. Magn. 30, 4740 (1994).

10. V. Bojarevics, A. Roy, and K. Pericleous, Magnetohydrodynamics 46, 317 (2010). 
11. V. Bojarevics and K. Pericleous, Fluid Mechanics and Its Applications, Vol. 80, ed. S. Molokov, R. Moreau, and H.K. Moffatt (New York: Springer, 2007), pp. 357-374.

12. L.D. Landau and E.M. Lifshitz, Electrodynamics of Continuous Media, 2nd ed. (Oxford, UK: Pergamon Press, 1984).

13. W.R. Smythe, Static and Dynamic Electricity (London, UK: McGraw-Hill, 1950).

14. R. Knauf, J. Piller, A. Seider, M. Stauber, and U. Zell, Proceedings of the 6th International Symposium Experimental Methods for Microgravity Materials Science, ed. R.A. Schiffman and J.B. Andrews (Warrendale, PA: TMS, 1994), pp. $43-51$.

15. G. Lohoefer and J. Piller (Paper presented at the 40th AIAA Aerospace Sciences Meeting \& Exhibit, AIAA 2002-0764, Monterey, CA, 2002).

16. R.W. Hyers and G. Trapaga, Solidification 1999, ed. S.P. Marsh, N.B. Singh, P.W. Voorhees, and W.H. Hofmeister (Warrendale, PA: TMS, 1999), pp. 23-32.

17. D.M. Matson, R.W. Hyers, T. Volkmann, and H.-J. Fecht, J. Phys. Conf. Ser. 327, 012009 (2011).
18. M.C. Flemings, D.M. Matson, W. Löser, R. Hyers, and J. Rogers, Science Requirements Document (SRD) for Levitation Observation of Dendrite Evolution in Steel Ternary Alloy Rapid Solidification (LODESTARS), NASA Document LODESTARS-RQMT-0001 (2003).

19. A.K. Gangopadhyay, R.W. Hyers, and K.F. Kelton, JOM (2012). doi:10.1007/s11837-012-0422-1.

20. G.M. Ludtka, Exploring Ultrahigh Magnetic Field Processing of Materials for Developing Customized Microstructures and Enhanced Performance, Final Technical Report (Oak Ridge, TN: Oak Ridge National Laboratory, 2005).

21. W.H. Hofmeister, C.M. Morton, R.J. Bayuzick, and M.B. Robinson, Solidification 1999, ed. S.P. Marsh, N.B. Singh, P.W. Voorhees, and W.H. Hofmeister (Warrendale, PA: TMS, 1999), pp. 75-82.

22. R.W. Hyers, G. Trapaga, and B. Abedian, Metall. Mater. Trans. B 34, 29 (2003).

23. R.W. Hyers, Meas. Sci. Technol. 16, 394 (2005). doi: 10.1088/0957-0233/16/2/010. 